\title{
Attitudes of primary school children towards the medical profession
}

\author{
Authors: Robert JH Sinnerton, ${ }^{A}$ Carina Bänziger, ${ }^{A}$ Frances Teasdale, ${ }^{B}$ James Hotham ${ }^{B}$ and Steven Backhouse ${ }^{B}$
}

\section{Aims}

The study aimed to assess the attitudes of primary school pupils towards the medical profession. The pupils were assessed before and after a clinical skills session aimed at teaching basic anatomy and practical skills. Primarily aimed at increasing the pupil's likelihood of considering a career in medicine, there was a secondary goal of education of basic life support.

\section{Methods}

Anonymous questionnaires were given to students before and after a 4-hour teaching session at the Multi-Profession Educational Centre in the Princess of Wales Hospital, Bridgend. The session was led by eight medical faculty comprised of six final-year medical students and two clinical teaching fellows. The questionnaires contained free-text response boxes and Likert scales. Word-clouds were generated to analyse free-text responses and a two-tailed significance test was used to analyse responses on the Likert scales.

\section{Results and conclusion}

All the pupils thoroughly enjoyed the teaching session. There was no negative impact upon their views on the medical profession or a career in medicine. There was a significant decrease in those that thought a career in medicine would be difficult (Fig 1). Although the data lacked power for significance, there was an increase in those wanting to be a doctor. Word-cloud analysis showed that after the session, pupils had a more rounded view of what doctors do as they showed an awareness of research and teaching, not just the clinical aspects. This novel teaching session aimed at primary school pupils made a career in medicine seem more achievable through a reduction in perceived difficulty. The session also served to address a lack of universal basic life support teaching across the UK with 24 pupils in Bridgend now familiar with cardiopulmonary resuscitation (CPR). Discussed are ideas for running further sessions like this and future work should consider analysing changes in healthcare related anxiety of some pupils.

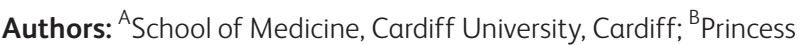
of Wales Hospital, Bridgend, UK

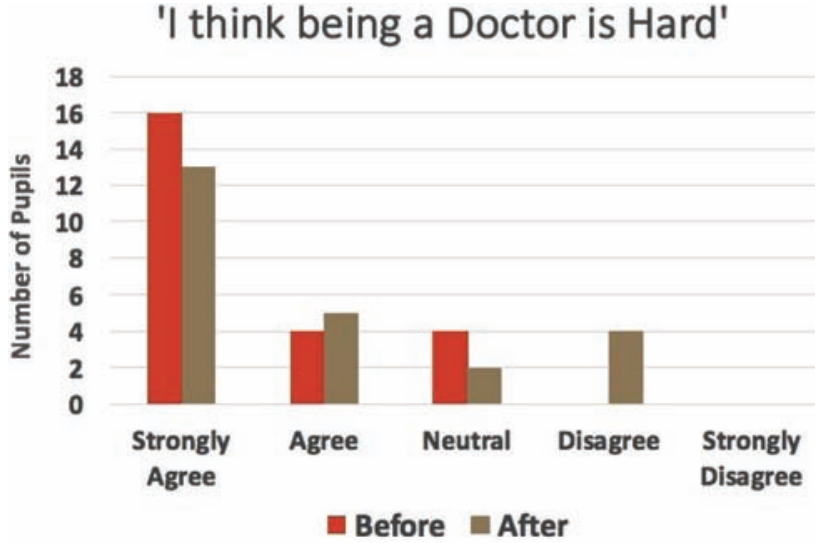

Fig 1. Results before and after the teaching session.

\section{Conflict of interest statement}

No conflicts of interest. 